\title{
EVALUACIÓN DE LA REPRODUCIBILIDAD DE DOS ÍNDICES ORIENTADOS A ANALIZAR LA HIGIENE DE LA LENGUA
}

\author{
${ }^{1}$ Iara Ballesteros G., ${ }^{1}$ Karen Ariadna Cobo C., ${ }^{1}$ Johen Patricia Navarro C., \\ ${ }^{2}$ Sonia Constanza Concha S. \\ ${ }^{1}$ Estudiante de X semestre F. Odontología U. Santo Tomás. ${ }^{2}$ Odontóloga, U. Santo Tomás, Especialista en Educación y Comunicación para la \\ Salud, Magíster en Epidemiología U. Industrial de Santander, Docente U. Santo Tomás.
}

Autor responsable de correspondencia: Karen Ariadna Cobo Cobo

Dirección de correo electrónico: kcobo20@hotmail.com

\section{RESUMEN}

Objetivo: Evaluar la reproducibilidad interevaluador de dos índices dirigidos a valorar la higiene de la lengua.

Materiales y métodos: Se realizó un estudio de evaluación de tecnología diagnóstica sobre una muestra de 100 personas que asistieron a las clínicas odontológicas de la Universidad Santo Tomás y en los que se tomó el índice de Miyazaki modificado analizando en forma cualitativa y cuantitativa. Para evaluar los niveles de reproducibilidad se aplicaron Test de Kappa ponderado y coeficiente de correlación de rangos de Sperman.

Resultados: Se registró mediante estadística de Kappa ponderada un coeficiente de 0.70 permitiendo evidenciar un buen nivel de reproducibilidad. Al analizar en forma cuantitativa el rango de Sperman alcanzó un nivel de reproducibilidad interevaluador de 0.83 .

Conclusión: El nivel de reproducibilidad interexaminador alcanzado por el índice modificado de Miyazaki que permite evaluar el nivel de higiene de la lengua de manera cualitativa y cuantitativa dio un resultado bueno y consistente.[Ballesteros I. Cobo KA, Navarro JP, Concha SC. Evaluación de la reproducibilidad de dos índices orientados a analizar la higiene de la lengua. Ustasalud Odontología 2008; 7: $35-41]$

Palabras claves: Cobertura de lengua, Higiene oral, Placa dental.

\section{REPRODUCIBILITY EVALUATION OF TWO INDICES ADDRESSED TO ANALYZE TONGUE HYGIENE}

\begin{abstract}
ASTRACT
Objetive: To evaluate inter-rater reproducibility of two indices addressed at assessing tongue hygiene.

Materials and Methods: A study about diagnostic technology evaluation was performed on a 100 subject sample who attended to dental clinics at Santo Tomas University. The modified Miyazaki's index was ranked in the subjects in a qualitative and quantitative mode. For assessing the reproducibility levels, the Kappa Test and Spearman rank correlation were used.

Results: The coefficient correlation was 0.70 ranked by Kappa statistics showing a good level of reproducibility. When analyzing the quantitative mode, Spearman rank inter-rater reproducibility level was0.83.

Conclusion: The inter-rater reproducibility level obtained by Miyazaki's modified index that allows for assessing the tongue hygiene in a qualitative and quantitative mode, showed it were good and consistent.
\end{abstract}

Key words: Tongue embracing, Oral hygiene, Dental plaque.

Recibido para publicación: 29 de marzo de 2008. Aceptado para publicación: 27 de junio de 2008.

\section{INTRODUCCIÓN}

Debido a su ubicación y función, la lengua es una de las estructuras anatómicas más importantes de la cavidad oral; ${ }^{1}$ está relacionada con otras estructuras bucodentales, más aún, está en estrecho contacto con los alimentos y con los microorganismos orales, por lo cual se pueden formar y observar depósitos de placa dentobacteriana sobre ésta. Las implica- ciones clínicas de estos cúmulos se han relacionado con enfermedades periodontales con la posibilidad de convertirse en una estructura intermediaria que favorece la halitosis y la transición de microorganismos a otras regiones del cuerpo, aspectos que alertan sobre la importancia de su higiene. ${ }^{2-5}$

La higiene oral se ha considerado como el mecanismo fundamental para preservar y promover la sa- 
lud bucodental. Sin embargo, ésta implica no sólo la higiene de los dientes, si no además de la lengua y tejidos adyacentes; ya que los estudios han demostrado que tiene gran importancia para el mejoramiento de la halitosis, en la prevención y control de la enfermedad periodontal y otras patologías presentes en cavidad oral. ${ }^{6-8}$

Sin embargo, la higiene de otras estructuras de cavidad oral se ha promovido poco; en ocasiones el odontólogo brinda escasa información y rara vez hace énfasis sobre la necesidad de la higiene de los tejidos blandos de la boca, incluyendo la lengua, hecho particularmente evidente en el caso de los adultos mayores. ${ }^{2,6}$

Por otra parte, tanto los odontólogos, como la población en general conocen la importancia de la adopción de medidas de higiene adecuadas; sin embargo, éstas aun no han alcanzado niveles óptimos que permitan en forma efectiva, alcanzar condiciones que favorezcan la prevención de las enfermedades bucodentales y fortalezcan la promoción de la salud a este nivel. ${ }^{7,8}$

El principal desafío que enfrentan hoy los odontólogos, es adecuar y concienciar a los niños, los adolescentes, los adultos y las personas mayores sobre la importancia de los hábitos de higiene oral adecuados. Desde como realizar un buen cepillado, no solo para mantener dientes sanos, sino también de los tejidos adyacentes, hasta llegar a garantizar un estado bucal o al menos conservarlo hasta la vejez, y para prevenir la halitosis, fenómeno que se ha constituido en una de las principales quejas de la mayoría de personas. Para esto se debe cuidar y conocer la estructura de la lengua, reconocer su estado, como debe realizarse el cepillado y el cuidado en general de esta estructura. $3,6,7,9$

Para la evaluación de la higiene de lengua, no se han encontrado en la literatura muchos índices; valdría la pena destacar el presentado por Miyazaki y colaboradores, y el de Miyazaki modificado que fue presentando por Winkel y también por Mantilla.,4 Para este último índice en particular, hay reportes de reproducibilidad en otros países. Sin embargo, en la literatura revisada no se encontraron artículos relacionados con la evaluación de los niveles de reproducibilidad de estos indicadores en Colombia y menos aun en Santander y la posibilidad de considerarlos como acciones básicas en el desarrollo de programas preventivos no parece visualizarse en un futuro cercano.

Al considerar lo ya expuesto, el objetivo de este estudio fue evaluar la reproducibilidad interevaluador de dos índices dirigidos a valorar la higiene de la lengua.

\section{MATERIALES Y METODOS}

Se realizó un estudio de evaluación de tecnología diagnóstica. ${ }^{10} \mathrm{El}$ universo estuvo conformado por pacientes que asistieron a la clínica odontológica de la universidad Santo Tomás de Floridablanca. La muestra la integraron 100 pacientes. Se consideraron como criterios de inclusión a los pacientes de ambos géneros que fueron atendidos en el área de rehabilitación y clínica integral de los semestres octavo, noveno y décimo, durante el primer semestre del 2007. Con edades entre 30 y 80 años. Como criterios de exclusión se definieron a las personas que registraron patologías a nivel de la lengua.

Para llevar a cabo esta investigación se diseñó una encuesta diligenciada por dos evaluadores previamente entrenados que incluía 15 preguntas que recopilaron variables sociodemográficas como edad, género, procedencia y otras variables relacionadas con los hábitos de higiene oral, consumo de bebidas oscuras, alcohólicas y hábitos de fumar. A estas preguntas, el encuestado debía responder Si o No según sus hábitos. A cada respuesta afirmativa se le dio un valor de 1 ; el valor fue 0 si era negativo. Anexo a las encuestas iban dos hojas, una con la grafica del índice de Miyazaki modificado para darle el valor de cobertura correspondiente a cada segmento de la lengua, y otra para los datos personales y el consentimiento del paciente.

Para la realización de esta actividad se solicitó la autorización a la Coordinación de las Clínicas Odontológicas, a cada paciente se le presentó el objetivo del trabajo y se obtuvo su consentimiento. Se registro la información general y finalmente, cada evaluador procedió a realizar el examen de higiene de lengua y a reportarla en forma cualitativa y cuantitativa.

Para el examen de la cobertura de la lengua, cada paciente se encontraba sentado en la unidad odontológica, se ubicó el plano de Frankfort paralelo al piso, se le solicitó que abriera ligeramente la boca y sacara la lengua lo máximo que pudiera. El evaluador se ubicó frente al paciente y al examinar el dorso de la lengua lo dividía en nueve segmentos, iniciaba en el segmento superior izquierdo y terminaba en el inferior derecho, cumpliendo con los criterios establecidos para el índice de Miyazaki modificado. Para observar la cobertura de lengua se estableció el modelo de Mantilla.,11 Cada segmento se calificó con valores que oscilaban entre 0 y 2 , en donde 0 representó ausencia de cobertura en el sector analizado, 1 cuando el color rosado de la lengua se observaba a través de la cobertura y 2 cuando la cobertura era total y no se observaba el color rosado a través de la ésta. Para describir el nivel de cobertura en forma cualitativa, se tomó 
en cada paciente el máximo valor obtenido y se registró ese como el nivel de cobertura de lengua registrado por el sujeto.

Para definir el nivel de cobertura en forma cuantitativa se sumaron los valores obtenidos en cada segmento se dividieron por nueve y se multiplicaron por 100.

Se efectuó una prueba piloto previa a la aplicación del instrumento que permitió verificar la comprensión y precisión de las preguntas, aspectos relacionados con el manejo de datos; se realizaron los cambios pertinentes y posteriormente se coordinó la aplicación de la encuesta.

Una vez obtenida las muestras se procedió a la sistematización de los datos en Excel y se exportó al paquete estadístico STATA 9.0 para el análisis correspondiente. ${ }^{12,13}$ Inicialmente, se describió el nivel de reproducibilidad interevaluador del índice de higiene de lengua analizado en forma cualitativa (Kappa ponderado) ${ }^{10}$ y cuantitativa (Coeficiente de Correlación de Sperman). ${ }^{14}$ Seguidamente se analizaron las variables sociodemográficas y compromisos sistémicos, de autocuidado oral, higiene de lengua, hábitos de consumo de las personas encuestadas según su nivel de cobertura de lengua, mediante test chi cuadrado y test exacto de Fisher. ${ }^{15,16}$ Se consideró un nivel de significancia $\alpha=0.05$.

Esta investigación se rigió dentro de los principios éticos establecidos en la resolución 008430 de 1993 del ministerio de protección social de la República de Colombia en los que se preservaron los derechos de autonomía beneficencia y justicia. ${ }^{17}$

\section{RESULTADOS}

\section{Nivel de reproducibilidad interevaluador}

El nivel de reproducibilidad registrado para el índice evaluado en forma cualitativa mediante la estadística Kappa ponderada registró un coeficiente de 0.70 que puede considerarse, de acuerdo al reportado por Landis y Koch, como substancial. ${ }^{18}$ De igual manera al analizarla en forma cuantitativa el coeficiente de correlación fue de 0.83 ; que anudado al análisis gráfico se hace evidente un buen nivel de reproducibilidad (Tabla 1, Figura 1).

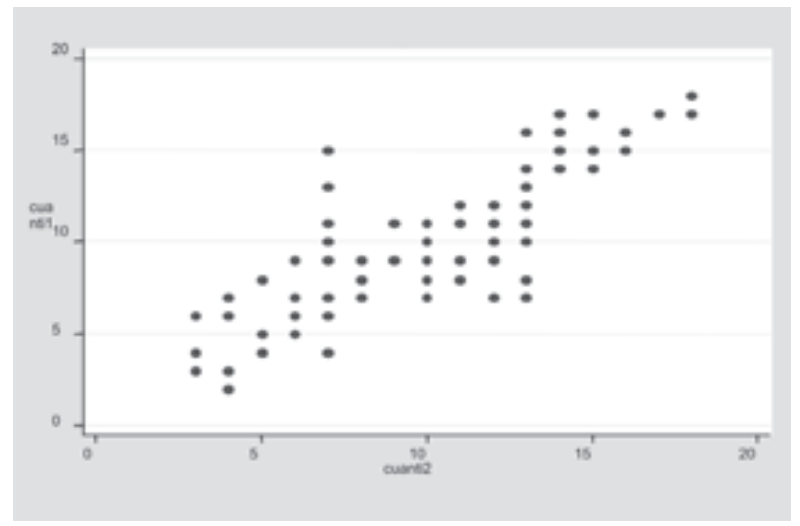

Figura 1. Análisis de la correlación interevaluador.

\section{Análisis univariado}

Se evaluaron un total de 100 personas, el 63\% eran mujeres, el 53\% estaban entre los 33 y 54 años de edad, el $77 \%$ procedían de la zona urbana y el $91 \%$ no reportaban compromisos sistémicos (Tabla 2).

Las variables relacionadas con autocuidado oral se evidenciaron en el 99\% de los sujetos. El 98\% de la población utilizaba la crema dental; el 33\% y $27 \%$ utilizan el enjuague y la seda dental, respectivamente (Tabla 3). En relación con la higiene de lengua, el $86 \%$ de la población reportó cepillarse la lengua y sólo el $31 \%$ haber recibido instrucciones por parte de un profesional sobre la higiene de ésta.

Las variables relacionadas con hábitos de consumo hacen evidente que el $85 \%$ de la población consume café, el $54 \%$ gaseosa o bebidas oscuras, un $23 \%$ toma alguna bebida alcohólica entre ellas se encontró que el 19\% consume cerveza. El 9\% fuma.

\section{Análisis bivariado}

No se reportaron asociaciones estadísticamente significativas de estas variables, según nivel de cobertura de la lengua. Sin embargo, es importante resaltar que $33(89.2 \%)$ hombres y 49 (77.8\%) mujeres registraban una cobertura gruesa. El 84.9\% (45) de las personas de 33 a 54 años tenían cobertura gruesa y el $83.5 \%$ (76) de las personas que no tenían compromiso sistémico registraban una cobertura gruesa de la lengua (Tabla 2).

Tabla 1. Descripción del nivel de reproducibilidad interevaluador del índice de higiene de lengua analizado en forma cualitativa y cuantitativa.

\begin{tabular}{lcc}
\hline \multicolumn{1}{c}{ ÍNDICE } & $\begin{array}{c}\text { Nivel de } \\
\text { reproducibilidad coeficiente }\end{array}$ & IC 95 \% \\
\hline Índice de higiene cualitativo & $0.70^{*}$ & - \\
Índice de higiene cuantitativo & $0.83^{* *}$ & $0.77-0.89$ \\
\hline
\end{tabular}

${ }^{*}$ Kappa ponderado ${ }^{* *}$ coeficiente de correlación de rangos de Sperman $\alpha=0.05$ 
Tabla 2. Descripción de variables sociodemográficas y sistémicas en la población evaluada y analizada en forma global y según nivel de cobertura de lengua.

\begin{tabular}{|c|c|c|c|c|}
\hline \multirow{2}{*}{ VARIABLE } & \multirow{2}{*}{$\begin{array}{l}\text { GLOBAL } \\
\text { Frec (\%) }\end{array}$} & \multicolumn{2}{|c|}{ NIVEL DE COBERTURA DE LENGUA } & \multirow{2}{*}{$\mathbf{P}^{*}$} \\
\hline & & $\begin{array}{l}\text { DELGADA } \\
\text { Frec (\%) }\end{array}$ & $\begin{array}{c}\text { Gruesa } \\
\text { Frec (\%) }\end{array}$ & \\
\hline Global & $100(100)$ & $18(18)$ & $82(82)$ & \\
\hline \multicolumn{5}{|l|}{ Género } \\
\hline Mujeres & $63(63)$ & $14(22,2)$ & $49(77.8)$ & $0.185^{*}$ \\
\hline Hombres & $37(37)$ & $4(10,8)$ & $33(89.2)$ & \\
\hline \multicolumn{5}{|l|}{ Edad } \\
\hline $33-54$ & $53(53)$ & $8(15.1)$ & $45(84.9)$ & $0.422^{* *}$ \\
\hline $55-88$ & $47(47)$ & $10(21.8)$ & $37(78.7)$ & \\
\hline \multicolumn{5}{|l|}{ Procedencia } \\
\hline Urbano & $77(77)$ & $12(15.6)$ & $65(84.4)$ & $0.250^{* *}$ \\
\hline Rural & $23(23)$ & $6(26.1)$ & 17 (73.9) & \\
\hline \multicolumn{5}{|l|}{ Compromiso sistémico } \\
\hline No & $91(91)$ & $15(16,5)$ & $76(83.5)$ & $0.203 *$ \\
\hline $\mathrm{Si}$ & $9(9)$ & $3(33.3)$ & $6(66.7)$ & \\
\hline
\end{tabular}

*Test Exacto de Fisher ${ }^{* *}$ Test de Chi cuadrado $\alpha=0,05$

Tabla 3. Descripción de la variable autocuidado oral en la población estudiada según el nivel de cobertura en lengua.

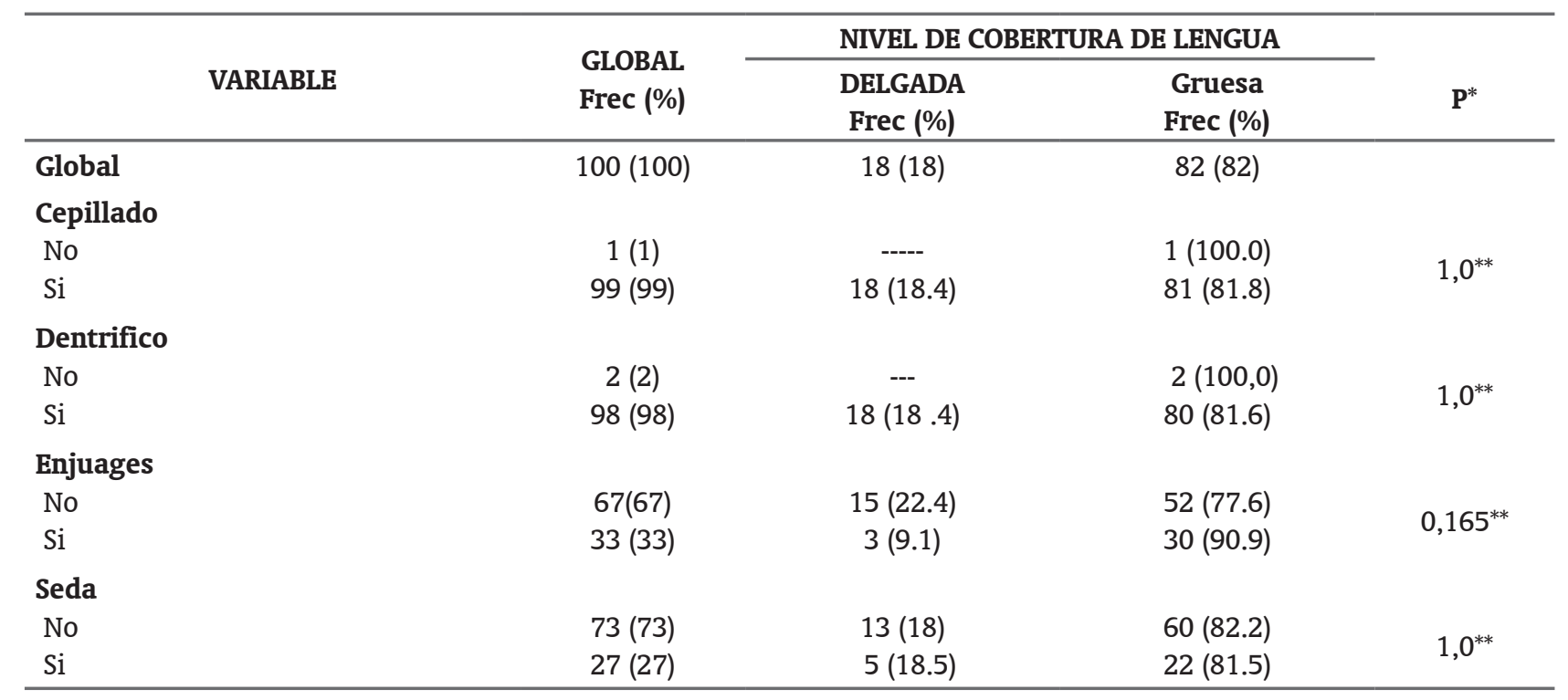

${ }^{*}$ Test de Fisher ${ }^{* *}$ Test de Chi cuadrado $\alpha=0,05$

Para las variables relacionadas con el autocuidado tampoco se observaron asociación estadísticamente significativa según cobertura de lengua. Es importante registrar que del total de personas que no utilizan cepillo y dentífrico el $100 \%$ de ellos registro cobertura gruesa; llama la atención que el $90.9 \%$ (30) de las personas que utilizan el enjuague tienen una cobertura gruesa.

Del total de personas que reportan que cepillan su lengua el $80.2 \%$ (69) tiene una cobertura gruesa. Del total de pacientes que reportan haber recibido instrucciones y el $77.4 \%$ (24) tienen una cobertura gruesa. No se repor- taron asociaciones estadísticamente significativas para estas variables analizadas (Tabla 4).

No se observaron asociaciones estadísticamente significativas para los variables hábitos de consumo según cobertura lengua. Sin embargo, cabe evidenciar que dentro del grupo de personas que consumen bebidas alcohólicas $82.6 \%$ (19) presentan cobertura gruesa; el 82\% (63) de las que no consumen bebidas alcohólicas presentan cobertura gruesa; también se observa, que del total de personas que reportan que fuman el $89 \%$ (8) presentan cobertura gruesa de la lengua (Tabla 5). 
Tabla 4. Descripción en personas evaluadas con higiene de lengua descritas según su cobertura.

\begin{tabular}{|c|c|c|c|c|}
\hline \multirow[b]{2}{*}{ VARIABLE } & \multirow{2}{*}{$\begin{array}{l}\text { GLOBAL } \\
\text { Frec (\%) }\end{array}$} & \multicolumn{2}{|c|}{ NIVEL DE COBERTURA DE LENGUA } & \multirow[b]{2}{*}{$\mathbf{P}^{*}$} \\
\hline & & $\begin{array}{c}\text { DELGADA } \\
\text { Frec (\%) }\end{array}$ & $\begin{array}{l}\text { Gruesa } \\
\text { Frec (\%) }\end{array}$ & \\
\hline \multicolumn{5}{|l|}{ Cepilla su lengua } \\
\hline No & $14(14)$ & $1(7.14)$ & $13(93)$ & \multirow{2}{*}{$0.455^{*}$} \\
\hline $\mathrm{Si}$ & $86(86)$ & $17(19.8)$ & $69(80.2)$ & \\
\hline \multicolumn{5}{|c|}{$\begin{array}{l}\text { Profesional indicación de cepillado } \\
\text { de lengua }\end{array}$} \\
\hline No & $69(69)$ & $11(16)$ & $58(84.1)$ & \multirow{2}{*}{$0.416^{* *}$} \\
\hline $\mathrm{Si}$ & $31(31)$ & $7(22.6)$ & $24(77.4)$ & \\
\hline
\end{tabular}

${ }^{*}$ Test exacto de Fisher ${ }^{* *}$ Test de Chi cuadrado $\alpha=0,05$

Tabla 5. Descripción de las variables relacionadas con hábitos de consumo analizadas en forma global y según nivel de cobertura de lengua.

\begin{tabular}{|c|c|c|c|c|}
\hline \multirow[b]{2}{*}{ VARIABLE } & \multirow[b]{2}{*}{$\begin{array}{l}\text { GLOBAL } \\
\text { Frec (\%) }\end{array}$} & \multicolumn{2}{|c|}{ NIVEL DE COBERTURA DE LENGUA } & \multirow[b]{2}{*}{$\mathbf{P}^{*}$} \\
\hline & & $\begin{array}{l}\text { Delgada } \\
\text { Frec (\%) }\end{array}$ & $\begin{array}{l}\text { Gruesa } \\
\text { Frec (\%) }\end{array}$ & \\
\hline \multicolumn{5}{|l|}{ Café } \\
\hline No & 15 (5) & $1(6.7)$ & $14(93,3)$ & \multirow{2}{*}{$0.295^{*}$} \\
\hline $\mathrm{Si}$ & $85(85)$ & $17(20.0)$ & $68(80.0)$ & \\
\hline \multicolumn{5}{|l|}{ Té } \\
\hline No & $90(90)$ & $16(18.0)$ & $74(82.2)$ & \multirow{2}{*}{$1.0^{*}$} \\
\hline $\mathrm{Si}$ & $10(10)$ & $2(20.0)$ & $8(80.0)$ & \\
\hline \multicolumn{5}{|l|}{ Gaseosa } \\
\hline No & $46(46)$ & 9 (19.6) & $34(80.4)$ & \multirow{2}{*}{$0,707^{* *}$} \\
\hline $\mathrm{Si}$ & $54(54)$ & $9(17)$ & $45(83.3)$ & \\
\hline \multicolumn{5}{|l|}{ Bebidas alcohólicas } \\
\hline No & $77(77)$ & 14 (18.1) & $63(82)$ & \multirow{2}{*}{$1,0^{*}$} \\
\hline $\mathrm{Si}$ & $23(23)$ & $4(17.3)$ & $19(82.6)$ & \\
\hline \multicolumn{5}{|l|}{ Vino } \\
\hline No & 97 (97) & 17 (17.5) & $80(82.4)$ & \multirow{2}{*}{$0,452^{*}$} \\
\hline $\mathrm{Si}$ & $3(3)$ & $1(33.3)$ & 2 (66.6) & \\
\hline \multicolumn{5}{|l|}{ Aguardiente } \\
\hline No & $92(92)$ & $17(18.4)$ & $75(81.5)$ & \multirow{2}{*}{$1,0^{*}$} \\
\hline $\mathrm{Si}$ & $8(8)$ & 1 (12.5) & 7 (87.5 ) & \\
\hline \multicolumn{5}{|l|}{ Cerveza } \\
\hline No & $81(81)$ & $15(18.5)$ & $66(81.4)$ & \multirow{2}{*}{$1,0^{*}$} \\
\hline $\mathrm{Si}$ & $19(19)$ & $3(16)$ & $16(84.2)$ & \\
\hline \multicolumn{5}{|l|}{ Fuma } \\
\hline No & $91(91)$ & 17 (19) & 74 (81.3) & \multirow{2}{*}{$1,0^{*}$} \\
\hline $\mathrm{Si}$ & $9(9)$ & $1(11.1)$ & $8(89)$ & \\
\hline
\end{tabular}

* Test Exacto de Fisher ${ }^{* *}$ Test de Chi cuadrado $\alpha=0.05$

\section{DISCUSIÓN}

Uno de los objetivos de este trabajo fue evaluar el nivel de reproducibilidad del índice modificado de cobertura lengua de Miyazaki y colaboradores. ${ }^{3}$ Es importante anotar, que éste debe ser el punto de partida para la implementación de cualquier tipo de programa orientado a promover la salud y prevenir las patologías bucodentales. El índice fue evaluado tanto en forma cualitativa como cuantitativa. Es necesario aclarar que el registro cuantitativo de este indicador fue una adaptación que se implementó para esta investigación. El propósito de hacerlo fue mostrar las potencialidades que pueden tener el considerar en forma integral la totalidad de la información recolectada. 
La evaluación cualitativa se ajustó a los estándares establecidos para el indicador, observándose consistencias en los resultados al evaluarse tanto en forma cualitativa como cuantitativa. De esta manera, se están abriendo alternativas que podrían facilitar la consolidación de resultados.

Al comparar estos resultados con los presentados por Mantilla y colaboradores, se observó que los niveles de reproducibilidad alcanzados entre los examinadores oscilaron entre 51\% y 64\%. ${ }^{3}$ Este último, relativamente comparable al registrado en esta investigación (0.7). En una investigación realizada por Gómez y colaboradores, el reporte de reproducibilidad para este mismo índice fue de 0.77 , similar a lo reportado en el presente trabajo.,11

Una de las mayores dificultades que surgieron en el análisis de los resultados, es la poca investigación relacionada con evaluación de la reproducibilidad del índice de cobertura de lengua reportada en la literatura, aspecto que puede mostrar el poco interés que existe frente a la posibilidad de implementar programas de promoción y prevención, más aún de adoptar mecanismos que favorezcan las estrategias de evaluación y dirigidas a promover la higiene de la lengua.

La lengua es el órgano más accesible, en estado normal y durante el reposo, la lengua ocupa la cavidad bucal. Puede ser considerada como órgano representante del aparato del gusto por su estructura esencialmente musculosa, desempeña un papel importante en la masticación, en la deglución, en la succión y por último en la articulación de determinados sonidos. ${ }^{1}$

La apariencia normal de la lengua tiene una capa delgada de una cobertura blanca; sin embargo, si la higiene no se efectúa de manera adecuada puede generar complicaciones de diversa índole y de ahí la importancia de promover la higiene de esta estructura. El estudio muestra que muchas personas no saben que la lengua también se debe cepillar para evitar la colonización de microorganismos que causan las diferentes patologías. Al referirse a la halitosis es importante retomar lo definido por Sterer y colaboradores, el mal aliento es una condición común, usualmente derivada de la microbiota oral. ${ }^{19}$ Los odontólogos no se han concientizado en la promoción sobre la higiene de la lengua, ni de los instrumentos adecuados para su higiene.

Kleinberg y Westbay, asociaron la halitosis con la reducción del suministro de oxígeno presente en el flujo salival que ocurre al levantarse; ${ }^{20}$ al haber un rápido flujo salival aumenta el oxigeno disponible lo cual disminuye la oportunidad de que los pépticos y las proteínas sean degradadas por las bacterias orales. ${ }^{8}$ Sin embargo, la reducción del flujo salival no tiene repercusiones exclusivas sobre la halitosis; también influye sobre la cantidad de depósitos que pueden llegar a acumularse en la lengua, lo que complica aún más el cuadro de halitosis que puede llegar a presentarse.

En este estudio, se evaluaron 100 personas de ambos géneros que acudieron a las clínicas odontológicas de Floridablanca, para la recolección de información se utilizó el índice de cobertura de lengua; para el examen clínico de la misma se aplicó el mismo que utilizó Mantilla y colaboradores., ${ }^{3,11}$ En éste, evaluaron los microorganismos presentes en cavidad oral. En contraste, en el presente trabajo no se intentó establecer relaciones con el conteo microbiano, relación con patógenos periodontales debido a las dificultades que existen para poder aislar y estudiar este tipo de microorganismos en nuestro medio; aspecto que podría considerarse como una limitación del presente trabajo.

Mantilla y colaboradores reportaron que un $61 \%$ de los sujetos entre las edades de 40 a 49 presentaron cobertura gruesa. ${ }^{3}$ García y colaboradores registraron una cobertura gruesa en 79 (86.8\%) en adultos mayores. ${ }^{21}$ Adicionalmente, en el estudio de Gómez y colaboradores, el nivel de cobertura gruesa se evidenció en el 88\% de los sujetos al inició de su estudio. ${ }^{8} \mathrm{Al}$ comparar estos resultados con el presente estudio, se observó que el $53 \%$ estaban en edades de 33 a 54 años con cobertura de lengua gruesa (84.9\%), lo que muestra la consistencia en los resultados e indica que la cobertura de lengua si parece aumentar con la edad.

Este aspecto también coincide con lo registrado en los estudios realizados por Rodríguez, García y López que sugieren que la cobertura de lengua aumenta con la edad. . $21,22^{2}$

Es importante anotar que en el estudio de Gómez y colaboradores se evidenció, además, la capacidad del índice de mostrar cambios en los niveles de cobertura de lengua después de implementar un programa orientado a promover la higiene de esta estructura y soporta aún más la importancia de sustentar la construcción de evidencia soportada en la existencia de índices validados. ${ }^{8}$

Es importante destacar que, los resultados de este trabajo indican la importancia del uso adecuado de los elementos de higiene oral y sobre cómo es probable que las personas con alta cobertura de lengua estén utilizando los enjuagatorios como un mecanismo para enmascarar la halitosis, pues del total de personas que utilizaban enjuagatorios más del 
$90 \%$ registraban cobertura gruesa en la lengua. Estos hallazgos coinciden con lo reportado en el estudio de Yaegaki y colaboradores, quienes indicaron que la halitosis deriva principalmente de la cobertura de la lengua de tal forma que es más importante la limpieza de ésta, más que el uso de enjuagues orales. $^{23}$

En el estudio de López y colaboradores, al igual que en este trabajo, se encontró que los hábitos de higiene bucodental en los adultos mayores son deficientes; ${ }^{22}$ aunque la mayoría de los individuos reportan que asean sus dientes y la lengua con una frecuencia diaria, al parecer, hay fallas en las técnicas de higiene pues fue posible observar gran cantidad de placa bacteriana. Lo anterior enfatiza la importancia de desarrollar programas de promoción y prevención en las personas mayores.

Rakowski y colaboradores sugieren que no es suficiente con poseer los conocimientos básicos y adecuados, de hábitos de autocuidado si no que además es importante reforzar motivar y sensibilizar a la población, para el mejoramiento no solo de su higiene oral si no mejorar la calidad de vida. ${ }^{24}$

Finalmente, Se podría concluir con este trabajo que el nivel de reproducibilidad registrado para el índice evaluado en forma cualitativa y cuantitativa fue bueno y que podría ser implementado para el monitoreo de programas promocionales y preventivos orientados a incentivar la higiene de la lengua.

\section{BIBLIOGRAFÍA}

1. Fehrenbach MJ, Herring SW. Anatomía de Cabeza y Cuello. Mc Graw - Hill Interamericana México DF; 1997.

2. Roldán S, Herrera D. Sanz M. Biofilms and the tongue: therapeutical approaches for the control of halitosis. Clin Oral Invest 2003; 7: 189 - 197.

3. Mantilla Gómez S, Danser MM, Sipos PM, Rowshani B, van der Velden U, van der Weijden GA. Tongue coating and salivarybacterial counts in healthy/gingivitis subjects and periodontitis patients. J Periodontol 2001; 28: 970 - 978.

4. Winkel EG, Roldán S, Van Winkelhoff AJ, Herrera D, Sanz M. Clinical effects of a new mouthrinse containing chlorhexidine, cetylpyridinium chloride and zinc-lactate on oral halitosis. J Clin Periodontol 2003; 30: 300 - 306.

5. Pedrazzi V, Sato S, de Mattos M, Lara E, Panzeri H. A comparative clinical Trial employing a toothbrush and a tongue scraper. J Periodontol 2004; 75: 1009 - 1012.

6. Ruiz-Medina P, Bravo M, Gil-Montoya JA, Montero J. Discrimination of functional capacity for oral hygiene in elderly Spanish people by the Barthel General Index. Community Dent Oral Epidemiol 2005; 33: 363 - 369.

7. Concha SC. Importancia de los índices que evalúan la higiene oral de prótesis dentales removibles en la implementación de programas promocionales y preventivos y criterios básicos para evaluar su reproducibilidad. Ustasalud Odontología 2005; 4: 38 - 43.
8. Gómez Y, Junca A, Gutiérrez DZ, Salazar L, Concha SC. Evaluación del impacto de un programa de higiene de lengua realizado en los centros geriátricos Centro del Bienestar del Anciano de Bucaramanga y el asilo Juan Pablo II de Floridablanca [Trabajo de Grado]. Bucaramanga: Universidad Santo Tomás; 2007.

9. Rodríguez M, Moreno L, Martínez CA. Factores orales asociados a halitosis en pacientes usuarios de prótesis total que asisten a las clínicas odontológicas de la universidad Santo Tomás. Ustasalud Odontología 2005; 4: 9 - 16.

10. Kraemer HC. Evaluating medical test. Objective and quantitative guidelines. Newbury Park Sage publications; 1992.

11. Danser MM, Gómez SM, van der Weijden GA. Tongue coating and tongue brushing: a literatura review. Int J Dent Hyg 2003; 1: $151-158$.

12. Microsoft Excel 5.0. NY. Microsoft Corporation, 1997.

13. Stata Corp. Stata Statistical Software. Release 9.0. College station. TX: Stata corporation 2005.

14. Williams A. How to...Write and analyses a questionnaire. J Orthod 2003; 30: 245 - 252,

15. Pagano M, Gauvreau K. Principles of Biostatistics. Belmont: Duxbury Press: 1995. p. 35 - 44, 257 - 263.

16. Norman GR, Streiner DL. Bioestadística. Madrid: Mosby/ Doyma Libros, 1996. p. 129-142.

17. República de Colombia. Ministerio de Salud. Resolución No. 008430 (Oct 4 1993) Bogotá.

18. Stata Corporations. Stata base reference manual. Vol 2 G-M Release 8. A STATA press Publications STATA publications. College Stations, Texas; 2003 p. 207-220.

19. Sterer N, Bar- Ness Greenstein R, Rosenberg M. Galactoside activity in saliva is associated with oral malodour. J Dent Res 2002; 81: 182 - 185.

20. Kleinberg I, Westbay G. Salivary and metabolic factors involved in oral malodour formation. J Periodontol 1992; 63: 768 - 775.

21. Garcia YM, Flórez LJ, Silva LM, Aguilar E, Concha SC. Evaluación de la efectividad de un programa educativo en higiene oral dirigido a cuidadores de adultos mayores de los asilos de San Antonio y San Rafael de la ciudad de Bucaramanga. Ustasalud 2006; 5: 40 - 48.

22. López C, Bejarano D, Jiménez DM, Damián Z, Morales LF, Calzada MT. Función gustativa en gerontes: comparación de la influencia de dos técnicas de higiene lingual en ancianos de cuatro hogares geriátricos de Cali. Revista Estomatológica 2005; 13: $20-29$.

23. Yaegaki K, Coil JM. Examination, classification, and treatment of halitosis, clinical perspectives. J Can Dent Assoc 2000; 66: 257- 261.

24. Rakowski W, Lang WP, Kerschubaum WE. Correlates of interest in dental health education with older adults: future perspective and quality interaction. Gerodontics 1987; 3: 191 - 197. 